\title{
IMPLEMETAÇÃO DA METODOLOGIA SAFETY HUDDLE NA TERAPIA INTENSIVA DURANTE A PANDEMIA COVID-19: RELATO DE EXPERIÊNCIA ${ }^{1}$
}

Lucas Rodrigo Garcia de Mello Barbara Pompeu Christovam ${ }^{1}$ Mylena da Cruz Araujo ${ }^{1}$ Ana Paula Amorim Moreira ${ }^{1}$ Erica Brandão de Moraes ${ }^{1}$ Graciele Oroski Paes ${ }^{3}$ Iuri Bastos Pereira ${ }^{4}$

\author{
http://orcid.org/0000-0002-4833-606X \\ http://orcid.org/0000-0002-9135-8379 \\ http://orcid.org/0000-0003-1939-7784 \\ http://orcid.org/0000-0003-1047-0658 \\ http://orcid.org/0000-0003-3052-158X \\ http://orcid.org/0000-0001-8814-5770 \\ http://orcid.org/0000-0002-6323-2883
}

Objetivo: relatar a implementação do Safety Huddle em unidades de terapia intensiva durante a pandemia da COVID-19 através de uma ferramenta eletrônica, desenvolvida por um grupo de enfermeiros intensivistas e profissionais da tecnologia da informação que atuam na saúde. Método: trata-se de um relato de experiência sobre a implementação da ferramenta Safety Huddle, através do método ágil Scrum. Resultados: a primeira etapa foi de identificação dos indicadores que deveriam ser incluídos para a realização do Safety Huddle nas Unidades de Terapia Intensiva e necessários ao monitoramento e discussão durante as reuniões de segurança. A segunda etapa foi de implementação do modelo proposto, com apresentação do fluxo de operacionalização da ferramenta Safety Huddle com envio diário do alerta intitulado "Alerta COVID-19". Conclusão: contribuiu para o gerenciamento e agilidade na identificação dos pacientes acometidos pela COVID-19, priorizando o atendimento de acordo com a condição clínica do paciente permitindo a disponibilidade dos leitos, otimização de recursos e assegurando os serviços assistenciais na instituição.

Descritores: Cuidados Críticos; Segurança do Paciente; Gestão de Riscos; Vírus da SARS; Qualidade em Saúde.

\section{IMPLEMETATION OF THE SAFETY HUDDLE METHODOLOGY IN INTENSIVE THERAPY DURING PANDEMIC COVID-19: EXPERIENCE REPORT}

Objective: to report the implementation of Safety Huddle in intensive care units during the COVID-19 pandemic through an electronic tool, developed by a group of intensive care nurses and information technology professionals working in health. Method: this is an experience report on the implementation of the Safety Huddle tool, using the agile Scrum method. Results: the first step was to identify the indicators that should be included for the Safety Huddle in the Intensive Care Units and necessary for monitoring and discussion during safety meetings. The second stage was the implementation of the proposed model, with the presentation of the operational flow of the Safety Huddle tool with daily dispatch of the alert entitled "COVID Alert-19". Conclusion: contributed to the management and agility in the identification of patients affected by COVID-19, prioritizing care according to the patient's clinical condition allowing the availability of beds, optimization of resources and ensuring assistance services in the institution.

Descriptors: Critical Care; Patient Safety; Risk Management; SARS Virus; Health Quality.

\section{IMPLEMETACIÓN DE LA METODOLOGÍA SAFETY HUDDLE EN TERAPIA INTENSIVA DURANTE PANDEMIC COVID-19: INFORME DE EXPERIENCIA}

Objetivo: informar la implementación de Safety Huddle en unidades de cuidados intensivos durante la pandemia COVID-19 a través de una herramienta electrónica, desarrollada por un grupo de enfermeras de cuidados intensivos y profesionales de tecnología de la información que trabajan en salud. Método: este es un informe de experiencia sobre la implementación de la herramienta Safety Huddle, utilizando el método ágil Scrum. Resultados: el primer paso fue identificar los indicadores que deberían incluirse para llevar a cabo Safety Huddle en las Unidades de Cuidados Intensivos y necesarios para el monitoreo y la discusión durante las reuniones de seguridad. La segunda etapa fue la implementación del modelo propuesto, con la presentación del flujo operativo de la herramienta Safety Huddle con el envio diario de la alerta titulada "Alerta 19 COVID". Conclusión: contribuyó al manejo y agilidad en la identificación de pacientes afectados por COVID 19, priorizando la atención de acuerdo con la condición clínica del paciente permitiendo la disponibilidad de camas, la optimización de recursos y asegurando servicios de asistencia en la institución.

Descriptores: Cuidados Críticos; Seguridad del Paciente; Gestión de Riesgos; Virus del SRAS; Calidad em Salud.

${ }^{1}$ O manuscrito é um desdobramento de um projeto de mestrado, submetido à Faculdade de Medicina da Universidade Federal Fluminense, com aprovação do CAAE no 17558819.9 .0000 .5243 e Parecer do CEP nㅇ 3.567.788.

${ }^{2}$ Universidade Federal Fluminense, Escola de Enfermagem Aurora Afonso Costa, Niterói, RJ.

${ }^{3}$ Universidade Federal Rio de Janeiro, Escola de Enfermagem Anna Nery, Rio de Janeiro, RJ.

${ }^{4}$ Universidade Federal do Rio de Janeiro, Curso de Enfermagem, Campus Professor Aloísio Teixeira, Macaé,RJ.

Autor Correspondente: Lucas Rodrigo Garcia de Mello E-mail: lucasgmello@ig.com.br

Recebido: $25 / 4 / 2020$

Aceito: $28 / 5 / 2020$ 


\section{INTRODUÇÃO}

A COVID-19 é uma infecção viral causado pelo SarsCoV-2 com manifestações clínicas graves que já infectou mais de 3.772 .367 de indivíduos, em aproximadamente 200 países e territórios, com a morte de mais de 244.239 indivíduos, segundo a Organização Mundial de Saúde (OMS) e a Johns Hopkins University ${ }^{(1-3)}$.

No Brasil, de acordo com o Ministério da Saúde (MS), já existem mais de 125.000 casos confirmados da doença, com mais de 8.500 óbitos resultando em uma taxa de letalidade de $6,8 \%$, entretanto, a região mais afetada é o Sudeste acumulando $46 \%$ dos $\operatorname{casos}^{(4)}$.

A evolução epidemiológica e o impacto da COVID-19 são incertos e existem dezenas de países com indivíduos apresentando sinais e sintomas graves, gerando uma sobrecarga aos cuidados de saúde e toda sua infraestrutura, impactando, principalmente, na capacidade operacional dos serviços, no que tange à alocação adequada de recursos materiais, equipamentos e capital humano, tanto quantitativa quanto qualitativamente, para atender a demanda aumentada de pacientes nas unidades de terapia intensiva (UTI), que requerem cuidados avançados devido à pandemia ${ }^{(5-6)}$.

De acordo com dados disponibilizados no site do Ministério da Saúde, até a primeira semana de maio de 2020, o Brasil possuía cerca de 33.795 leitos instalados de terapia intensiva adulto classificados como: Leitos UTI adulto do Sistema Único de Saúde (SUS), Leitos UTI adulto não SUS e Leitos UTI habilitados ${ }^{(7)}$.

Contudo, frente aos índices crescentes de casos confirmados e de casos graves da doença, os serviços hospitalares precisam incorporar estratégias e ferramentas que propiciem uma gestão eficiente dos fluxos assistenciais e, possibilitem o enfrentamento dos desafios que o sistema de saúde brasileiro vem apresentando, ao longo dos anos, e que se agravaram com a pandemia da COVID-19. Estas são: aumento da demanda de cuidados avançados em decorrência da complexidade clínica da doença; capacidade instalada de leitos de UTI diminuida; tempo de permanência elevado na UTI; escassez de insumos (principalmente equipamentos de proteção individual), equipamentos (ventiladores) e profissionais; aumento dos custos assistenciais; e, por conseguinte, garantir a prestação de cuidados seguros e de qualidade ${ }^{(3-7)}$.

Frente a esse contexto, o Conselho Federal de Enfermagem (Cofen) reforça a importância da Resolução no 543/17 para nortear os gestores na alocação do capital humano, considerando que ela estabelece os parâmetros mínimos de dimensionamento do quadro de profissionais com base no perfil do serviço de saúde e de enfermagem e no grau de dependência do paciente(8)

Portanto, no cenário de uma UTI um dos maiores desafios dos gestores de enfermagem é garantir ações de cuidado seguras ao paciente, considerando a alocação adequada dos profissionais de enfermagem de acordo com as necessidades de assistência direta e indireta dos pacientes. Porém, esse desafio se torna ainda mais crítico nos pacientes acometidos pela COVID-19, pois necessitam de suportes de alta complexidade para que os desfechos sejam positivos, ou seja, cura ${ }^{(8-10)}$.

Sendo assim, os enfermeiros, que atuam nas unidades de terapia intensiva, utilizam o Nursing Activities Score (NAS) para o monitoramento diário de sua carga de trabalho requerida pelos pacientes críticos, garantindo - dimensionamento adequado dos profissionais e otimizando a alocação de recursos, pois a qualidade da assistência de enfermagem é um fator contribuinte com alto impacto para gerar desfechos favoráveis com excelentes resultados ${ }^{(9-10)}$.

No entanto, as UTI necessitam estabelecer os critérios de admissão e alta dos pacientes. Por isso, o Conselho Federal de Medicina (CFM) publicou a Resolução no 2.156/16 com o objetivo de estabelecer esses critérios nas UTI. Segundo a resolução, as admissões na UTI devem ser baseadas em diagnóstico e necessidade do paciente, serviços médicos disponíveis na instituição, priorização de acordo com a condição do paciente, disponibilidade de leitos e potencial benefício para o paciente com as intervenções terapêuticas e prognóstico (11-12).

Ademais, corroborando comas resoluções supracitadas, três pontos são cruciais e determinantes para garantir a eficiência na gestão do fluxo de pacientes, principalmente durante a pandemia: a garantia da disponibilidade dos leitos, a priorização da internação de acordo com a condição clínica do paciente e por fim assegurar os serviços assistenciais na instituição. Portanto, a comunicação efetiva é fundamental para assegurar gestão operacional adequada ${ }^{(5,11-13)}$.

Neste contexto da comunicação efetiva, otimização de recursos e diminuição de riscos relacionados à assistência multidisciplinar, surge a metodologia intitulada Safety Huddle, também chamada de "reunião de segurança" proposta pelo Institute Healthcare Improvement (IHI). Esse método aumenta a conscientização de segurança no nivel operacional, ou ainda, da linha de frente e ajuda a organização a desenvolver uma cultura de segurança $a^{(14)}$.

No entanto, para operacionalizar e garantir o sucesso do método, alguns indicadores foram eleitos para o monitoramento do paciente na terapia intensiva: número de leitos disponiveis; número de internações; taxa de 
ocupação; giro de rotatividade; intervalo de substituição; taxa de utilização de ventilação mecânica (VM); tempo médio de VM; carga de trabalho pelo Nursing Activities Score (NAS) alta; e óbito. As instituições já possuíam esses indicadores para a gestão da terapia intensiva, porém não estavam focadas nos subgrupos de pacientes acometidos pela COVID-19(8-10).

Conhecer a variabilidade no fluxo do paciente é, definitivamente, necessário, mas não é suficiente para resolver esse problema atual de maneira adequada. Entendemos ser necessário estabelecer um time de profissionais da instituição, envolvendo as áreas (administrativas e assistenciais) para garantia da operacionalização adequada do fluxo de pacientes na instituição(5-11).

Dessa forma, um grupo de hospitais independentes manifestou a necessidade de adaptar uma ferramenta de gestão que já possuía o Safety Huddle para garantir, o mais precocemente possivel, a obtenção das informações referentes ao paciente com COVID-19 e aos recursos disponiveis para garantir o atendimento no momento oportuno. Foi sugerido, por um time de enfermeiros especialistas em terapia intensiva e que atuam como consultores na área de Qualidade e Segurança do Paciente, a implementação do Safety Huddle nas UTI com o foco nos pacientes acometidos pela doença.

Com isso, esse estudo teve como objetivo relatar a experiência na implementação do Safety Huddle em unidades de terapia intensiva, durante a pandemia da COVID-19, através de uma ferramenta eletrônica, desenvolvida por um grupo de enfermeiros intensivistas e profissionais da tecnologia da informação que atuam na saúde.

\section{MÉTODO}

\section{Tipo de estudo}

Trata-se de um estudo descritivo do tipo relato de experiência sobre a implementação do Safety Huddle em UTI com foco na pandemia COVID 19.

\section{Cenário do estudo}

O estudo teve como cenário 8 hospitais privados, distribuídos em diferentes regiões do Brasil, que já utilizavam um software para o gerenciamento de indicadores na terapia intensiva.

\section{Periodo de realização da experiência}

A adaptação do sistema eletrônico do Safety Huddle e a implementação com foco para a pandemia da COVID-19 foi realizado no período de 16 de março a 5 abril de 2020

\section{Sujeitos envolvidos na experiência}

Gestores de qualidade e segurança do paciente, gestores das UTI, consultores enfermeiros com expertise na área e profissionais de tecnologia da informação.

\section{Aspectos éticos}

É um desdobramento de um projeto de mestrado que foi submetido à Faculdade de Medicina da Universidade Federal Fluminense, com aprovação do Certificado de Apresentação para Apreciação Ética (CAAE) no 17558819.9.0000.5243 e Parecer do Comitê de Ética em Pesquisa (CEP) nㅇ 3.567.788. A título de divulgação da iniciativa, requisitou-se previamente a autorização da empresa responsável pelo software. Por se tratar de um relato de experiência, foi solicitada a autorização prévia da diretoria da empresa responsável pelo software para divulgação da iniciativa. De acordo com o que preceitua a Resolução n 466/12 do Conselho Nacional de Ética em Pesquisa (CONEP), nenhum dado e/ou informação que identificasse os hospitais e os participantes envolvidos foi revelado.

\section{DESCRIÇÃO DA EXPERÊNCIA}

Devido à pandemia da COVID-19 no Brasil, recebemos a solicitação de um grupo de aproximadamente 8 hospitais privados, distribuidos em diferentes regiões do Brasil que já utilizavam um software para o gerenciamento de indicadores na terapia intensiva. A necessidade apontada pelo grupo era de reunir todas as informações relacionadas ao fluxo de pacientes, recursos e desfecho dos pacientes acometidos pela COVID-19 e garantir uma comunicação efetiva e imediata referente à temática.

Dessa forma, os gestores sugeriram ao time de enfermeiros consultores de uma empresa, com expertise na área, que propusesse a adaptação na ferramenta para a identificação precoce dos casos suspeitos e confirmados com doença, utilização de recursos, carga de trabalho da enfermagem, comunicação e discussão multidisciplinar com as equipes da instituição.

Entretanto, para elaborar o Safety Huddle com o foco na COVID-19 foram operacionalizadas as seguintes etapas:

- Construção do Safety Huddle com o foco na pandemia COVID-19: revisão da produção científica disponível na literatura, a fim de mapear o conhecimento sobre a temática, assim como, a identificação dos indicadores utilizados para gestão clínica das UTI e incorporação de novos recursos para aperfeiçoar o conhecimento.

- Implementação do Safety Huddle nas UTI apresentação da ferramenta para as equipes envolvidas, definição do fluxo para operacionalização do Safety Huddle na prática. 
Foi definida, pela equipe dos enfermeiros e profissionais da TI, a utilização do método ágil Scrum para elaboração da ferramenta e implementação da ferramenta nas instituições de saúde.

\section{PRINCIPAIS RESULTADOS ALCANÇADOS}

\section{Etapa 1 - Construção do Safety Huddle com o foco na pandemia COVID-19}

Considerando o modelo proposto pelo IHI para o Safety Huddle, entendemos a necessidade dos hospitais em operacionalizar a metodologia voltada para um subgrupo específico de pacientes.

A primeira ação do projeto se deu através de uma reunião com gestores médicos e de enfermagem das UTI com a participação dos lideres de qualidade e segurança do paciente dos hospitais que já utilizavam um software para gestão dos indicadores. Devido à pandemia e as medidas de isolamento social, essa reunião foi realizada por videoconferência através do software Google Hangouts da versão 80.0.3987.163

Para esse primeiro momento, tínhamos pouco tempo de reunião devido à alta demanda nas instituições, utilizamos duas ferramentas de qualidade, a saber: brainstorming, que se trata de uma técnica para estimular a criatividade em grupo, e que pode ser traduzida como "tempestade de ideias", através dessa técnica foi possivel identificar quais indicadores eram necessários para realizar o monitoramento; e a discussão durante as reuniões de segurança. Os indicadores definidos estão descritos no Quadro 1:

Quadro 1 - Indicadores identificados para o Safety Huddle

\begin{tabular}{|c|c|c|}
\hline Fluxo de Pacientes & Recursos & Desfechos \\
\hline $\begin{array}{l}\text { Número de leitos destinados } \\
\text { para à COVID-19 }\end{array}$ & $\begin{array}{l}\text { Uso de suporte } \\
\text { ventilatório não } \\
\text { invasivo }\end{array}$ & Alta \\
\hline $\begin{array}{l}\text { Número de internações por } \\
\text { suspeita da COVID-19 e } \\
\text { casos confirmados } \\
\text { Número de internações com } \\
\text { SARI (Síndrome de Infecção } \\
\text { Respiratória Aguda Grave) }\end{array}$ & $\begin{array}{l}\text { Uso de } \\
\text { ventilação } \\
\text { mecânica (VM) } \\
\text { invasiva }\end{array}$ & \\
\hline Taxa de ocupação & $\begin{array}{l}\text { Tempo médio } \\
\text { de VM }\end{array}$ & Óbito \\
\hline Giro de rotatividade & \multirow{3}{*}{$\begin{array}{l}\text { Carga de } \\
\text { trabalho da } \\
\text { Enfermagem } \\
\text { (NAS) }\end{array}$} & \\
\hline $\begin{array}{l}\text { Tempo médio de } \\
\text { permanência }\end{array}$ & & \\
\hline Intervalo de substituição & & \\
\hline
\end{tabular}

Atualmente o time de desenvolvimento do software é composto por três enfermeiros especialistas em terapia intensiva e gestão em saúde, dois profissionais de tecnologia da informação com formação em desenvolvimento e programação e um gerente de produto, também enfermeiro, especialista na área de qualidade e segurança do paciente. A metodologia utilizada, pelo time de desenvolvimento, para construção do software é o Scrum.

Segundo alguns autores, o Scrum é definido como uma metodologia que agiliza e otimiza a gestão e o planejamento de projetos de software. A utilização do método gera benefícios como: melhoria na comunicação entre as equipes de trabalho; aumento na satisfação das equipes que desenvolvem produtos e serviços e melhoria da qualidade dos produtos e serviços elaborados ${ }^{(15)}$.

Após o levantamento dos indicadores, discutimos a operacionalização através da elaboração de um fluxo na ferramenta Bizzagi Modeler versão 3.6 para realizar uma validação com a equipe de tecnologia de informação. $\bigcirc$ fluxo de trabalho foi apresentado para os membros da equipe que discutiram os requisitos e a estimativa de horas para o desenvolvimento do modelo.

$\mathrm{Na}$ metodologia Scrum preconiza-se que seja criado um plano de trabalho descrevendo todas as ações e/ou funcionalidades a serem desenvolvidas, considerando uma prática importante para a organização e gerenciamento dos requisitos coletados cuja responsabilidade é compartilhada com o time de desenvolvimento(15).

O Safety Huddle foi construido em um período de três semanas e, para divulgação e apresentação do modelo, desenvolveu-se um plano remoto de comunicação para os gestores, devido às restrições de mobilidade.

\section{Etapa 2 - Implementação do Safety Huddle nas UTI}

Foi realizada uma reunião, por videoconferência, com os hospitais envolvidos no projeto para apresentação do fluxo de operacionalização da ferramenta Safety Huddle.

O fluxo consistia em, diariamente, enviar um alerta intitulado "Alerta COVID-19" por e-mail às 06h00min da manhã com o status atual da unidade de terapia intensiva para um time de profissionais constituido pela instituição. Através do e-mail era possível acessar um painel com todas as informações necessárias para discussão do Safety Huddle. O painel era atualizado a cada 2 minutos, pois era integrado com os dados do sistema administrativo do hospital e o software utilizado para gestão dos indicadores. 
Figura 1 - Protótipo do "Alerta COVID-19"

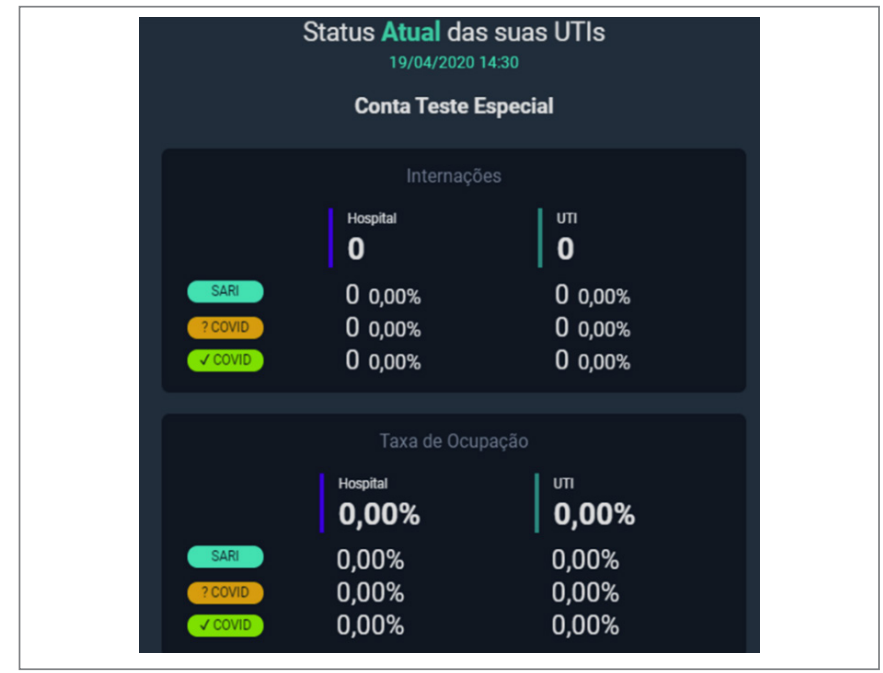

Além dos indicadores definidos para o monitoramento das UTI na perspectiva dos pacientes com COVID-19, as equipes do núcleo de segurança do paciente $e$ controle de infecção hospitalar eram responsáveis por complementar a discussão através de informações que corroboravam com a compreensão do cenário e permitir uma tomada decisão mais assertiva. São elas: dados relacionados a exame laboratorial, mapa de precauções especiais e incidentes relacionados ao desabastecimento, equipamentos biomédicos, principalmente, envolvendo ventilador mecânico até às 07h00min com objetivo de detectar precocemente quaisquer problemas ou condições inseguras na linha de cuidado do paciente crítico. As 08h00min da manhã era realizada a reunião chamada de Stand Up Meeting, também conhecida como "reunião em pé ou de piso", para conseguir maior agilidade e não prejudicar a rotina, quase em colapso, da UTI.

Nessa reunião, a partir dos resultados da análise dos indicadores e discussão com as informações do Núcleo de Segurança do Paciente (NSP) e Controle de Infecção Hospitalar $(\mathrm{CCIH})$, era realizada uma análise situacional com objetivo de otimizar o fluxo e o plano terapêutico dos pacientes nas UTI. Com isso, o objetivo era identificar, através da busca ativa, os pacientes que já poderiam receber cuidados intermediários, ou seja, pacientes que já estavam sem o uso da ventilação mecânica, estabilidade hemodinâmica, porém, requerendo avaliações médicas e de enfermagem diariamente pelo alto risco de precisarem de intervenção imediata. Assim sendo, o Safety Huddle permitiu a liberação do leito mais precoce possivel para um novo paciente, garantindo a priorização na admissão dos pacientes de acordo com a gravidade da doença.

Para facilitar a compressão das etapas do processo, inserimos o fluxo para operacionalização do Safety Huddle.

Figura 2 - Fluxo do Safety Huddle

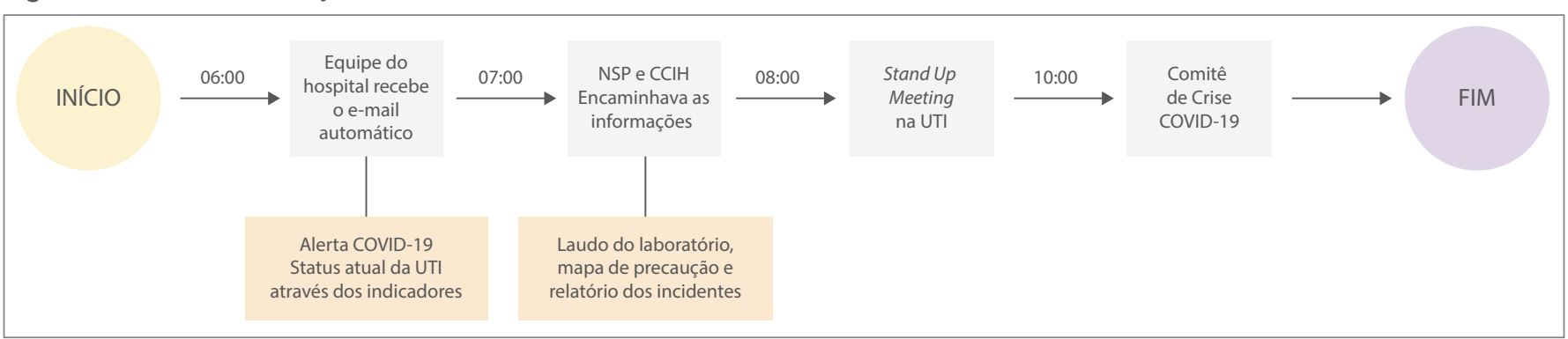

E por último era realizado um encontro às 10h00min da manhã, intitulada Comitê de Crise, cujo objetivo era reportar para alta liderança executiva das instituições, o cenário da instituição, as dificuldades e os entraves apontados pelas equipes para prestar uma assistência segura aos pacientes acometidos pela doença e validar as ações discutidas nos planos de ação; além de empoderar os profissionais da linha de frente, promover um ambiente seguro para os profissionais de saúde e articular de esforços necessários para enfrentar esse importante desafio.

\section{Limitações da Experiência}

Destaca-se como uma importante limitação do estudo, o pouco tempo para explorar outros dados e informações para automatizar ainda mais o processo de trabalho e a impossibilidade de implementar, presencialmente, o fluxo nas UTI devido às medidas de restrição e isolamento social. Além disso, o relato descreve apenas a utilização do modelo na UTI durante a pandemia.

\section{Contribuições para a prática}

O estudo contribuiu para a melhoria e otimização do fluxo de pacientes nas UTI através da comunicação efetiva entre os profissionais, além de destacar o papel do enfermeiro na criação de ferramentas e recursos tecnológicos para aperfeiçoar a gestão do cuidado nas mesmas.

\section{CONSIDERAÇÕES FINAIS}

O objetivo do estudo foi contemplado, pois a implementação do Safety Huddle permitiu agilizar a 
identificação dos pacientes acometidos pela COVID-19, priorizando o atendimento de acordo com a condição clínica do paciente permitindo a disponibilidade dos leitos, otimização de recursos e assegurando os serviços assistenciais na instituição. Porém, reconhece que a comunicação efetiva, definida como uma meta internacional de segurança do paciente, e o trabalho da equipe multidisciplinar são compreendidos como fatores determinantes para garantia da qualidade e segurança do paciente nas instituições de saúde.

Com o avanço da doença e a dificuldade de compreender a sua história natural, faz-se necessária a realização de novas pesquisas com outros métodos para consolidar a importância da ferramenta na gestão clínica das UTI.

CONTRIBUIÇÃO DOS AUTORES: a) concepção e/ou desenho do estudo: Lucas Rodrigo Garcia de Mello e Mylena da Cruz Araujo; b) coleta, análise e interpretação dos dados: Lucas Rodrigo Garcia de Mello; Mylena da Cruz Araujo e Ana Paula Amorim Moreira; c) redação e/ou revisão crítica do manuscrito: Graciele Oroski Paes; Érica Brandão de Moraes e Barbara Pompeu Christovam; d) aprovação da versão final a ser publicada: Barbara Pompeu Christovam e Iuri Bastos Pereira.

\section{REFERÊNCIAS}

1. World Health Organization (WHO). Coronavirus disease (COVID-19) dashboard [Internet]. Geneva: WHO [updated 2020 May 07; cited 2020 May 07]. Available from: https://COVID19.who.int/ 2. Johns Hopkins University \& Medicine, Coronavirus Resource Center. COVID-19 Case Tracker [Internet]. Baltimore: JHU [update daily; cited 2020 May 07]. Available from: https://coronavirus.jhu.edu/

3. Coronavirus: novel coronavirus (COVID-19) infection. Elsevier [Internet]. 2020 Feb 5 [updated 2020 Apr 17; cited 2020 Apr 23]. Available from: https://www.elsevier.com/__data/assets/ pdf_file/0010/977698/novel-coronavirus-COVID-19-infection-2020-04-17.pdf

4. Coronavirus Brasil [Internet]. [updated 2020 Apr 22; cited 2020 Apr 22]. Brasilia: Ministério da Saúde. Available from: https://COVID.saude.gov.br/. Portuguese.

5. Emanuel EJ, Persad G, Upshur R, Thome B, Parker M, Glickman A, et al. Fair Allocation of Scarce Medical Resources in the Time of COVID-19. N Engl J Med. 2020. doi: 10.1056/NEJMsb2005114.

6. Chen N, Zhou M, Dong X, Qu J, Gong F, Han Y, et al. Epidemiological and clinical characteristics of 99 cases of 2019 novel coronavirus pneumonia in Wuhan, China: a descriptive study. Lancet [Internet]. 2020 [cited 2020 Apr 22];395(10223):507-13. Available from: https://www.thelancet.com/action/showPdf?pii=S0140-6736\%2820\%2930211-7

7. Ministério da Saúde (BR), Sala de Apoio à Gestão Estratégica (SAGE). Painel de Leitos e Insumos [Internet]. Brasília: Ministério da Saúde [cited 2020 May 07]. Available from: https://COVID-insumos.saude.gov.br/paineis/insumos/painel.php. Portuguese.

8.Conselho Federal de Enfermagem (BR). Resolução no 543 de 18 de abril de 2017. Atualiza e estabelece parâmetros para o Dimensionamento do Quadro de Profissionais de Enfermagem nos serviços/locais em que são realizadas atividades de enfermagem. Diário Oficial [da] República Federativa do Brasil, Brasilia, DF, n. 86, p. 120, 8 mai. 2017. Seção 1. Available from: http://www.cofen. gov.br/resolucao-cofen-5432017_51440.html. Portuguese.

9. Batassini E, Silveira JT, Cardoso PC, Castro DE, Hochegger T,
Vieira DFVB, et al. Nursing Activities Score: what is the ideal periodicity for assessing workload? Acta paul enferm [Internet]. 2019 Mar [cited 2020 Apr 18];32(2):162-8. Available from: http:// www.scielo.br/pdf/ape/v32n2/en_1982-0194-ape-32-02-0162. pdf

10. Conz CA, Aguiar RS, Reis HH, Pinto MCJ, Mira VL, Merighi MAB. Atuação de enfermeiros lideres de unidade de terapia intensiva: abordagem compreensiva. Enferm Foco [Internet]. 2019 [cited 2020 Apr 18];10(4):41-6. Available from: http://revista.cofen.gov.br/index.php/enfermagem/article/view/2196/603. Portuguese

11. Ministério da Saúde (BR), Agência Nacional de Vigilância Sanitária. Resolução no 7, de 24 de fevereiro de 2010. Dispõe sobre os requisitos mínimos para funcionamento de Unidades de Terapia Intensiva e dá outras providências [Internet]. Diário Oficial [da] República Federativa do Brasil, Brasilia, DF, n. 37, p. 48, 25 fev. 2010. Seção 1. Available from: https://www.saude.mg. gov.br/images/documentos/RDC-7_ANVISA\%20240210.pdf. Portuguese.

12. Conselho Federal Medicina. Resolução $n=2.156$, de 28 de outubro de 2016 Estabelece os critérios de admissão e alta em unidade de terapia intensiva [Internet]. Diário Oficial [da] República Federativa do Brasil, Brasília, DF, p. 138-139, 17 de nov. 2016. Seção I Available from: https://sistemas.cfm.org.br/normas/visualizar/resolucoes/BR/2016/2156. Portuguese.

13. Felipe TRL, Spiri WC. Construção de um instrumento de passagem de plantão. Enferm Foco [Internet]. 2020 [cited 2020 Apr 22];10(7):76-82. Available from: http://revista.cofen.gov.br/ index.php/enfermagem/article/view/2451/553.Portuguese.

14. Institute for Healthcare Improvement. Huddles [Internet]. London: IHI; 2004 [cited 2020 May 07]. Available from: http:// www.ihi.org/resources/Pages/Tools/Huddles.aspx

15. Carvalho BV, Mello CHP. [Implementation of scrum agile methodology in software product project in a small technology-based company]. Gest Prod. 2012;19(3):557-73. doi: 10.1590/ S0104-530X2012000300009. Portuguese. 